\title{
REMARKS ON BODENMILLER'S THEOREM
}

Oswald Giering zum 60 . Geburtstag gewidmet

Rudolf Fritsch

The original version of BODENMILLER's Theorem states that the three circles with the diagonals of a complete quadrilateral as diameters intersect in the same two points. We provide a simple analytic proof of this fact which yields a much more general result. Furthermore we discuss some special configurations appearing in this context.

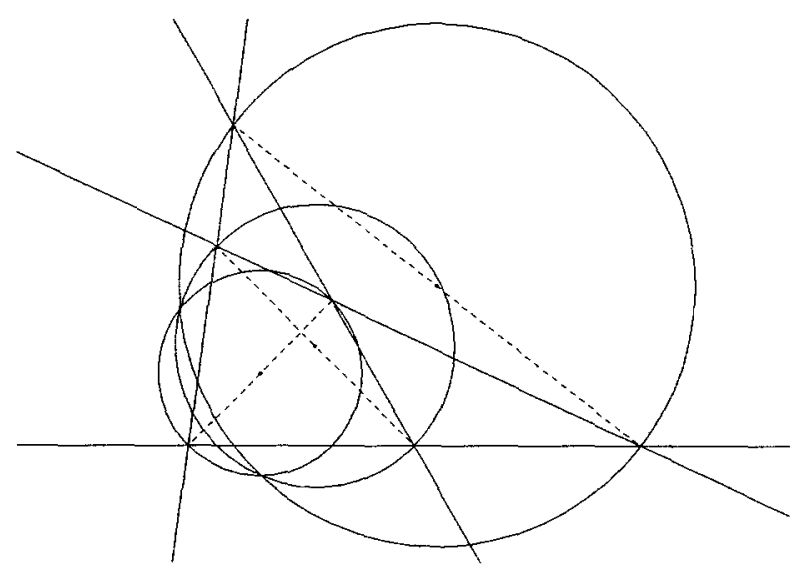

The historical background and the classical proofs of BODENMILLER's theorem using the standard theorems of synthetic geometry (theorems of APOLLONIUS, MENELAUs, MONGE and GAuss, Theorem of the Complete Quadrilateral) are described in [2] while an approach via descriptive geometry has been given by G. WEISS [7]. Here we treat the subject analytically. 
Let $V$ be a vector space over a field $K$ with char $K \neq 2$ and let a non-degenerate, symmetric bilinear form

$$
<,>: V \times V \longrightarrow K,(\boldsymbol{x}, \boldsymbol{y}) \mapsto<\boldsymbol{x}, \boldsymbol{y}>=\boldsymbol{x} \cdot \boldsymbol{y}
$$

be given. The form $\langle,>$ determines a set of affine quadrics in $V$ : For each $\boldsymbol{m} \in V$ and $c \in K$ we have the set

$$
Q=Q_{\boldsymbol{m}, c}=\left\{\boldsymbol{x} \in V \mid \boldsymbol{x}^{2}-2 \boldsymbol{m} \cdot \boldsymbol{x}+c=0\right\}
$$

which is called an sphere if it contains at least two elements. The point ${ }^{1} \boldsymbol{m}$ is called the center of $Q$ and the value of the function

$$
P=P_{\boldsymbol{m}, c}: V \longrightarrow K, \boldsymbol{x} \mapsto \boldsymbol{x}^{2}-2 \boldsymbol{m} \cdot \boldsymbol{x}+c
$$

at place $\boldsymbol{x} \in V$ is the power of $\boldsymbol{x}$ with respect to $Q$; thus, a sphere is the zero set of its power function. We denote by $\mathcal{Q}$ the set of the spheres determined by the fixed form $<,>$.

In case $K=\mathbb{R}$, the field of reals (or more generally, if $K$ is an ordered field in which every positive element is a square), and $V=K^{2}$ we have central conics. The following special cases are worthy of note:

- If $\langle$,$\rangle is the standard scalar product, \mathcal{Q}$ contains circles only. This situation yields the original version of BODENMILLER's theorem.

- If $\langle$,$\rangle is a (positive or negative) definite form, \mathcal{Q}$ consists of similar ellipses with parallel axes ${ }^{2}$. The corresponding generalization of BODENMILLER's theorem is due to C. Gudermann.

- If $\langle$,$\rangle is an indefinite form { }^{3}, \mathcal{Q}$ is a set of hyperbolas with parallel asymptotes. The validity of BODENMILLER's theorem for these spheres has been noted by G. PICKERT.

We return to the general situation. A 2 -set (= unordered pair) $\{\boldsymbol{u}, \boldsymbol{v}\}$ of points in $V$ is called a diameter of the sphere $Q \in \mathcal{Q}$ with center $\boldsymbol{m}$ if both points belong to the sphere and $\boldsymbol{m}$ is their midpoint, $\mathrm{ie}^{4}$,

$$
\boldsymbol{m}=\frac{1}{2}(\boldsymbol{u}+\boldsymbol{v})
$$

The basis for any version of BODENMILLER's theorem lies in the following fact.

\footnotetext{
${ }^{1}$ If there is no danger of misunderstanding we use the notions "vector" and "point" synonymously.

${ }^{2}$ Compare the definition of a metric by an ellipse in PICKERT [5, Section 5.1].

${ }^{3}$ That is the pseudoeuclidean case in the terminology of LENZ [4, p. 317]; see also the discussion "metric by a hyperbola" in Pickert [5, Section 5.2].

${ }^{4}$ Here the assumption on the characteristic of the ground field becomes essential.
} 
PROPOSITION 1. For any 2-set $\{\boldsymbol{u}, \boldsymbol{v}\} \subset V$ with $\boldsymbol{u} \neq \boldsymbol{v}$ there is exactly one sphere $Q \in \mathcal{Q}$ with $\{\boldsymbol{u}, \boldsymbol{v}\}$ as a diameter of $Q$, namely,

$$
Q_{u, v}=\{\boldsymbol{x} \in V \mid(\boldsymbol{x}-\boldsymbol{u}) \cdot(\boldsymbol{x}-\boldsymbol{v})=0\}
$$

Proof. Let $Q_{\boldsymbol{m}, c}$ a sphere with $\{\boldsymbol{u}, \boldsymbol{v}\}$ as diameter. From $2 \boldsymbol{m}=\boldsymbol{u}+\boldsymbol{v}$ we obtain

$$
\begin{aligned}
P_{\boldsymbol{m}, c}(\boldsymbol{x}) & =\boldsymbol{x}^{2}-2 \boldsymbol{m} \cdot \boldsymbol{x}+c= \\
& =\boldsymbol{x}^{2}-(\boldsymbol{u}+\boldsymbol{v}) \cdot \boldsymbol{x}+c= \\
& =(\boldsymbol{x}-\boldsymbol{u}) \cdot(\boldsymbol{x}-\boldsymbol{v})-\boldsymbol{u} \cdot \boldsymbol{v}+c
\end{aligned}
$$

Therefore

$$
P_{\boldsymbol{m}, c}(\boldsymbol{u})=0 \Leftrightarrow c=\boldsymbol{u} \cdot \boldsymbol{v}
$$

and thus, $Q_{\boldsymbol{u}, \boldsymbol{v}}$ is the unique sphere with the 2 -set $\{\boldsymbol{u}, \boldsymbol{v}\}$ as a diameter.

We call $Q_{\boldsymbol{u}, \boldsymbol{v}}$ the sphere over the 2 -set $\{\boldsymbol{u}, \boldsymbol{v}\}$. In the sequel we write $P_{\boldsymbol{u}, \boldsymbol{v}}$ instead of $P_{\boldsymbol{m}, \mathrm{c}}$ for the power function of the sphere $Q_{u, v} ;$ it takes the form

$$
\boldsymbol{x} \mapsto(\boldsymbol{x}-\boldsymbol{u}) \cdot(\boldsymbol{x}-\boldsymbol{v})=\boldsymbol{x}^{2}-(\boldsymbol{u}+\boldsymbol{v}) \cdot \boldsymbol{x}+\boldsymbol{u} \cdot \boldsymbol{v}
$$

Next, we have to consider quadrilaterals. A (complete) quadrilateral in $V$ is a set of four lines ( = one-dimensional affine subspaces of $V$ ) such that every two intersect but no three are concurrent ${ }^{5}$. Since up to now we have no restriction on $\operatorname{dim} V$, it is worthwhile to note that any complete quadrilateral is contained in an affine subplane of $V$. To see this, observe that two of the four lines determine an affine plane since they intersect and, since each of the other two lines has two points in common with this plane, the quadrilateral lies completely therein.

The lines constituting a quadrilateral are called sides of the quadrilateral while the intersection of two sides is called a vertex. Each vertex is opposite to a (unique) second one (if both are not joined by a side) resulting in three such pairs for each quadrilateral. These 2 -sets constitute the diagonals of the quadrilateral. We specialize this general theory by naming the spheres over the diagonals BODENMILLER spheres of the quadrilateral.

In order to that the statement of the generalized BODENMILLER theorem, to follow, will be crystal clear, we recall the behaviour of spheres in $\mathcal{Q}$ under intersection. To this end let

\footnotetext{
${ }^{5}$ Here we adapt the definition in $[1, p, 231]$ for projective planes to our affine situation
} 
two spheres $Q_{1}, Q_{2} \in \mathcal{Q}$ be given with different centers $\boldsymbol{m}_{1}, \boldsymbol{m}_{2}$ and let $P_{1}, P_{2}$ denote their respective power functions. Then the intersection $Q_{1} \cap Q_{2}$ is contained in the zero set of the function $P_{1}-P_{2}$. The latter set, called the radical axis of $Q_{1}, Q_{2}[1$, p. 86], is an affine hyperplane of $V$ since the form $\langle$,$\rangle is assumed to be non-degenerate. Furthermore, this$ set is orthogonal to the line connecting the centers $\boldsymbol{m}_{1}, \boldsymbol{m}_{2}$ (with respect to the form $<,>$ ) and is the locus of points of equal power with respect to the two spheres.

At this point, we examine two special cases which fall out of the original statement of BoDENMILLER's theorem. Firstly, the BODENMILLER circles of a quadrilateral may touch each other in just one point (with the common tangent as radical axis of each pair of BODENMILLER circles); secondly, they may be pairwise disjoint (see [2]). For these cases, the notion of that the two circles "intersect in the same two points" may dealt with purely algebraically by counting zeros of multiplicity 2 twice and of allowing complex roots of the associated quadratic equations. Another possibility is to separate the claim in different cases:

- the BodenMiller circles intersect in the same two points;

- if two BoDENMILLER circles are tangent then all three are tangent at the same point;

- if two Bodenmiller circles are disjoint, then all three are pairwise disjoint.

The latter could be summarized as "the intersection of all three BODENMILLER circles of a quadrilateral is equal to the intersection of any two of them" which is evidently a weaker statement than the former. This difficulty could be resolved by establishing the claim

the three radical axes coincide

or, equivalently,

the power functions of the three BODENMILLER circles of a quadrilateral are linearly dependent in the vector space of all functions $V \rightarrow K$.

Both of the previously stated BODENMILLER's theorems would then be immediate consequences of this assertion.

We have now laid the necessary background for the statement of the main theorem.

THEOREM 2. The three radical axes formed by the BODENMILLER spheres of a quadrilateral, taken pairwise, coincide.

For the proof we may assume one vertex of the quadrilateral being the origin $\boldsymbol{O}$ of $V$ and consider the diagonal $\{\boldsymbol{a}, \boldsymbol{c}\}$ as forming a linearly independent set of vectors in $V$. Then we 
have scalars $r, s \in K$ with $r, s \neq 0, r, s, r s \neq 1$ such that $\{r a, s c\}$ is another diagonal and we can compute the vertex $b$ opposite to $O$ obtaining

$$
\boldsymbol{b}=\frac{r s-r}{r s-1} \boldsymbol{a}+\frac{r s-s}{r s-1} \boldsymbol{c} .
$$

This gives for the power functions of the BODENMILLER spheres

$$
(r s-1) P_{\boldsymbol{O}, \boldsymbol{b}}=r s P_{\boldsymbol{a}, \boldsymbol{c}}-P_{r \boldsymbol{a}, \boldsymbol{s} \boldsymbol{c}}
$$

showing their linear dependence and thus, the theorem.

The given statement of this theorem also takes into account the fact that hyperbolas might degenerate to intersecting pairs of lines and that the intersection of two such degenerate hyperbolas might be a complete line. We provide an example. Take $K=\mathbb{R}, V=\mathbb{R}^{2}$ and

$$
\left(x_{1}, x_{2}\right) \cdot\left(y_{1}, y_{2}\right)=x_{1} y_{1}-x_{2} y_{2}
$$

for all $\boldsymbol{x}=\left(x_{1}, x_{2}\right), \boldsymbol{y}=\left(y_{1}, y_{2}\right) \in V$. Consider a quadrilateral comprised of the axes and the lines determined by the equations

$$
\begin{aligned}
x_{1}+2 x_{2} & =1 \\
2 x_{1}+x_{2} & =1
\end{aligned}
$$

The BODENMILLER conics of this quadrilateral are now the degenerate hyperbolas given by the equations

$$
\begin{aligned}
& \left(x_{1}-x_{2}\right)\left(x_{1}+x_{2}-\frac{1}{3}\right)=0 \\
& \left(x_{1}-x_{2}\right)\left(x_{1}+x_{2}-\frac{1}{2}\right)=0 \\
& \left(x_{1}-x_{2}\right)\left(x_{1}+x_{2}-1\right)=0
\end{aligned}
$$

whose intersection is the diagonal $\{(x, x) \in V \mid x \in \mathbb{R}\}$, the radical axis of each pair of them.

The main theorem enables us to define the radical axis of a complete quadrilateral as the radical axis of two of its BODENMILLER spheres. Under the assumptions of the proof of the main theorem, it has the equation

$$
[(r-1) \boldsymbol{a}+(s-1) \boldsymbol{c}] \cdot \boldsymbol{x}=(r s-1) \boldsymbol{a} \cdot \boldsymbol{c} .
$$

In what follows we restrict our attention to the case with $\operatorname{dim} V=2$ so that the spheres are central conics and the radical axis of a quadrilateral is a line. By our definition this line is 
the locus of all points having the same power with respect to the three Bodenmiller circles of the quadrilateral. But it can be also seen as a locus from another point of view which we are going to describe in the sequel. For the presentation, we recall the notion of orthogonal affinity with axis $g$; that is a mapping $A: V \rightarrow V$ satisfying the following properties

1. the points of the line $g$ are fixed;

2. collinear points are mapped on collinear points;

3. the vector $A(c)-c$ is orthogonal to the line $g$, for any point $c \in V$.

Thus, an orthogonal affinity is a either a central collineation (with centre on the line at infinity) or an orthogonal projection of $V$ onto a line in $V$. The mapping $A$ is determined by the axis $g$ and the image $A(c)$ of one point $c \notin g$. An explicit description of $A$, needed later, is obtained by assuming the origin to lie on the axis $g$ and considering a further point $\boldsymbol{a} \in \boldsymbol{g}$. If, in addition, we set $\boldsymbol{n}=A(\boldsymbol{c})-\boldsymbol{c}$, then the vectors $\boldsymbol{a}, \boldsymbol{c}$ form a basis of $V$ and

$$
A(x \cdot \boldsymbol{a}+y \cdot \boldsymbol{c})=x \cdot \boldsymbol{a}+y \cdot(\boldsymbol{c}+\boldsymbol{n})
$$

for all $x, y \in K$.

The property of the radical axis of a quadrilateral referred to above may now be stated as follows.

THEOREM 3. Let $g$ be a side of a complete quadrilateral and let $\boldsymbol{b}, \boldsymbol{c}, \boldsymbol{d}$ denote the vertices of the triangle formed by the remaining sides; further let $A$ be an orthogonal affinity with axis $g$. Then the perpendiculars from the images $A(\boldsymbol{b}), A(\boldsymbol{c}), A(\boldsymbol{d})$ to the corresponding sides of the quadrilateral concur at a point of the radical axis (see figure). By a suitable choice of A, every point on the radical axis of the quadrilateral can be obtained in this way.

Before proving this theorem we mention some special cases. If $A$ is the identity mapping on $V$ then the theorem tells that the orthocentres of the triangles obtained by removing just one side of the quadrilateral belong to the radical axis. The fact that these orthocentres are collinear has already been noted by J. STEINER [2]. At the other extreme end, if $A$ is the orthogonal projection of $V$ onto $g$ then the point obtained is called the orthopole of $g$ with respect to the triangle $\boldsymbol{b}, \boldsymbol{c}, \boldsymbol{d}$. It is known that the four resulting orthopoles and the named orthocentres are collinear, see R. GoORMATIGH [3], consequently, the radical axis of the quadrilateral is also referred to as the eight-point-line of the quadrilateral [6, page 83]. Note that the orthocentre of a triangle always exists while there is no orthopole of a line if the line is isotropic. 


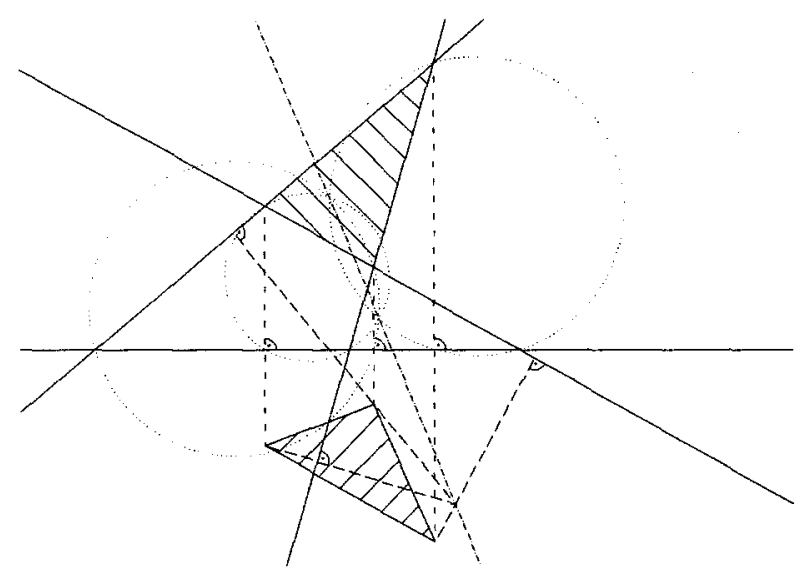

For the proof we describe the vertices of the quadrilateral by vectors as in the proof of the main theorem. We choose $g$ as the side consisting of the scalar multiples of $\boldsymbol{a}$, then $\boldsymbol{b}, \boldsymbol{c}$ and $\boldsymbol{d}=s \cdot \boldsymbol{c}$ are the vertices of the triangle to consider. Since we already have $A(\boldsymbol{c})-\boldsymbol{c}=\boldsymbol{n}$ we easily compute the quantities

$$
\begin{aligned}
& A(\boldsymbol{b})=\boldsymbol{b}+\frac{r s-s}{r s-1} \cdot \boldsymbol{n} \\
& A(\boldsymbol{d})=s \cdot(\boldsymbol{c}+\boldsymbol{n}) .
\end{aligned}
$$

Now the perpendiculars through the points $A(\boldsymbol{b}), A(\boldsymbol{c}), A(\boldsymbol{d})$ to the corresponding sides of the triangle $\boldsymbol{b}, \boldsymbol{c}, \boldsymbol{d}$ are the zero sets of the linear functions

$$
\begin{aligned}
L_{\boldsymbol{b}}(\boldsymbol{x}) & =\boldsymbol{c} \cdot(\boldsymbol{x}-A(\boldsymbol{b})) \\
L_{\boldsymbol{c}}(\boldsymbol{x}) & =(s \boldsymbol{c}-\boldsymbol{a}) \cdot(\boldsymbol{x}-A(\boldsymbol{c})), \\
L_{\boldsymbol{d}}(\boldsymbol{x}) & =(\text { ra }-\boldsymbol{c}) \cdot(\boldsymbol{x}-A(\boldsymbol{d}))
\end{aligned}
$$

The relation $(r s-1) \cdot L_{\boldsymbol{b}}=r \cdot L_{\boldsymbol{c}}+L_{\boldsymbol{d}}$ shows that these lines concur and, since the radical axis of the quadrilateral is the zero set of the function $L_{\boldsymbol{c}}+L_{\boldsymbol{d}}$, the point of concurrence belongs to the radical axis.

It remains to show that each point of the radical axis is obtained in this way, which in turn, requires a solution $\boldsymbol{n}$ for the system

$$
\begin{array}{r}
a \cdot \boldsymbol{n}=0 \\
(s c-a) \cdot(x-c-n)=0
\end{array}
$$

of equations, given a point $\boldsymbol{x}$ of the radical axis. Since the given bilinear form is nondegenerate by hypothesis, such a solution exists and is uniquely determined. 
Finally we specialize to the real case with the standard scalar product where the question arises as to the classification of the complete quadrilaterals with respect to how the BODENMILLER circles are related with respect to intersection. We offer some preliminary results concerning some conditions for the appearance of tangent BODENMILLER circles.

An immediate case occurs when two diagonals of the quadrilateral are parallel which causes the centers of all BODENMILLER circles to lie on a line incident with the third diagonal. This implies that the touching point also lies on this diagonal and so it must be one of the vertices on the line. It now follows from the famous theorem on the angle in the half circle - sometimes attributed to THALES of Milete - that the sides of the quadrilateral which intersect at this vertex must be perpendicular to each other.

To obtain a more general situation for tangent circles, we may start with any such pair with different centers. Next, we choose a diameter $\left\{\boldsymbol{u}_{1}, \boldsymbol{v}_{1}\right\}$ of one circle and a diameter $\left\{\boldsymbol{u}_{2}, \boldsymbol{v}_{2}\right\}$ of the other such that neither the connecting lines of $\boldsymbol{u}_{1}, \boldsymbol{u}_{2}$ and $\boldsymbol{v}_{1}, \boldsymbol{v}_{2}$ nor the connecting lines of $\boldsymbol{u}_{1}, \boldsymbol{v}_{2}$ and $\boldsymbol{v}_{1}, \boldsymbol{u}_{2}$ are parallel. These four named lines now form a quadrilateral with touching BODENMILLER circles.

This construction can still be generalized somewhat. We start with one circle and a vector $\boldsymbol{m}_{2}$, different from the center, and look for a second circle with center $\boldsymbol{m}_{2}$ tangent to the first chosen circle. There are two possibilities: if $\boldsymbol{m}_{2}$ belongs to the first circle, then there is just one choice for the second circle; otherwise there are two. This situation is algebraically treated in the appendix of [2].

In [2], one also finds a discussion of the case where one fixes the vectors $\boldsymbol{O}, \boldsymbol{a}, \boldsymbol{c}$ and the scalar $s$, see the proof of the main theorem. To find a scalar $r$ such that a quadrilateral with touching BODENMILLER circles is obtained is equivalent to the construction of the midpoint $\boldsymbol{m}_{3}$ of $\{r \boldsymbol{a}, s \boldsymbol{c}\}$. This is, in turn, an intersection point of

1. the line parallel to the line connecting $O, a$ through $\frac{1}{2}(a+s c)$ and

2. the hyperbola, the locus of the points whose distances from $\frac{1}{2}(\boldsymbol{a}+\boldsymbol{c}), s \boldsymbol{c}$ have the difference $\frac{1}{2}(a-c)$.

To achieve a quadrilateral with such tangent BODENMILLER circles would seem to be a nice exercise for a ruler and compass construction.

At this point we leave a further treatment open for future research and, in closing, thank G. Pickert for a fruitful correspondence on the subject, R. Eddy for a careful reading and correction of the manuscript. 


\section{REFERENCES}

[1] COXETER, H. S. M.: Introduction to Geometry, New York / London / Sidney / Toronto: John Wiley \& Sons ${ }^{2} 1969$

[2] FRITSCH, R.: Gudermann, Bodenmiller und der Satz von Bodenmiller - Steiner, in: Didaktik der Mathematik 20, 165 - 187 (1992)

[3] GOORMATHTIGH, R.: Question 2388, in: Nouvelles Annales de Mathématiques, Série 4, 19, 39 (1919)

[4] LENZ, H.: Grundlagen der Elementarmathematik, München / Wien: Carl Hanser Verlag ${ }^{3} 1976$

[5] PICKERT, G.: Metrische Geometrie in vektorieller Darstellung, Stuttgart: Klett 1983

[6] SCHRÖDER, E. M.: Geometrie euklidischer Ebenen, Paderborn: Schöningh 1985

[7] WEIB, G.: Eine räumliche Deutung der Vierseiteigenschaft von Bodenmiller, in: Elemente der Mathematik 37, 21 - 23 (1982)

Rudolf Fritsch

Mathematisches Institut

Ludwig-Maximilians-Universität

Theresienstraße 39

W-8000 München 2

Germany

Eingegangen am 23. April 1992; in revidierter Form ari 15. März 1993 